\section{UJMM

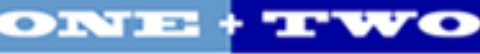

Volume 2 | 2009 Fall

\section{Undergraduate Journal of Mathematical}

Modeling: One + Two

2009

\title{
That's a Drag: The Effects of Drag Forces
}

Shane Maxemow

University of South Florida

Advisors:

Arcadii Grinshpan, Mathematics and Statistics

Karim Nohra, Civil \& Enviromental Engineering

Problem Suggested By: Karim Nohra

Follow this and additional works at: https://digitalcommons.usf.edu/ujmm

Part of the Mathematics Commons

UJMM is an open access journal, free to authors and readers, and relies on your support:

Donate Now

\section{Recommended Citation}

Maxemow, Shane (2009) "That's a Drag: The Effects of Drag Forces," Undergraduate Journal of Mathematical Modeling: One + Two: Vol. 2: Iss. 1, Article 4.

DOI: http://dx.doi.org/10.5038/2326-3652.2.1.4

Available at: https://digitalcommons.usf.edu/ujmm/vol2/iss1/4 


\title{
That's a Drag: The Effects of Drag Forces
}

\begin{abstract}
Drag is a force that opposes motion due to an object's shape, material, and speed. This project defined what drag force is, derived the governing equation for drag and listed some applications of drag forces. Derivation of the drag equation was achieved using the Buckingham $\pi$ theorem, a dimensional analysis tool. Lastly, this project explored the problem of how long and how far a dragster takes to stop once its parachute is deployed.
\end{abstract}

\section{Keywords}

Drag Force, Buckingham's Theorem, Drag Coefficient

\section{Creative Commons License}

\section{(c) (i) (3)}

This work is licensed under a Creative Commons Attribution-Noncommercial-Share Alike 4.0 License. 


\section{TABLE OF CONTENTS}

Problem Statement...............................................................................................

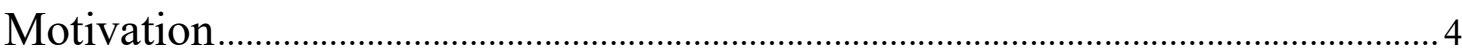

Mathematical Description and Solution Approach ..................................................... 4

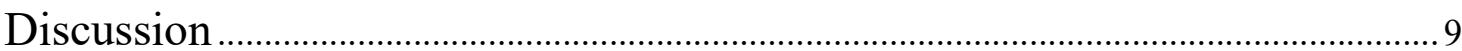

Conclusion and Recommendations....................................................................... 10

Nomenclature

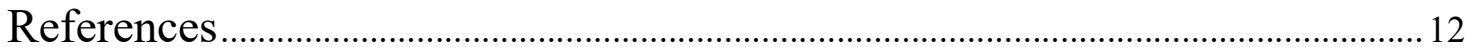

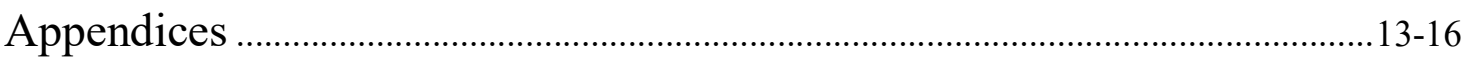




\section{PROBLEM STATEMENT}

This project seeks to achieve three main goals:

1. Define what drag forces are

2. Derive the standard drag equation

3. Utilize the drag equation to solve the following example:

A $600-\mathrm{kg}$ dragster is traveling with a velocity of $125 \mathrm{~m} / \mathrm{s}$ when the engine is shut off and the braking parachute is deployed. If air resistance imposed on the dragster due to the parachute is given by the function $F_{D}=\left(6000+0.9 v^{2}\right) \mathrm{N}$ where $v$ is in $\mathrm{m} / \mathrm{s}$, determine the time required for the dragster to come to rest. Also find the distance traveled in that time period.

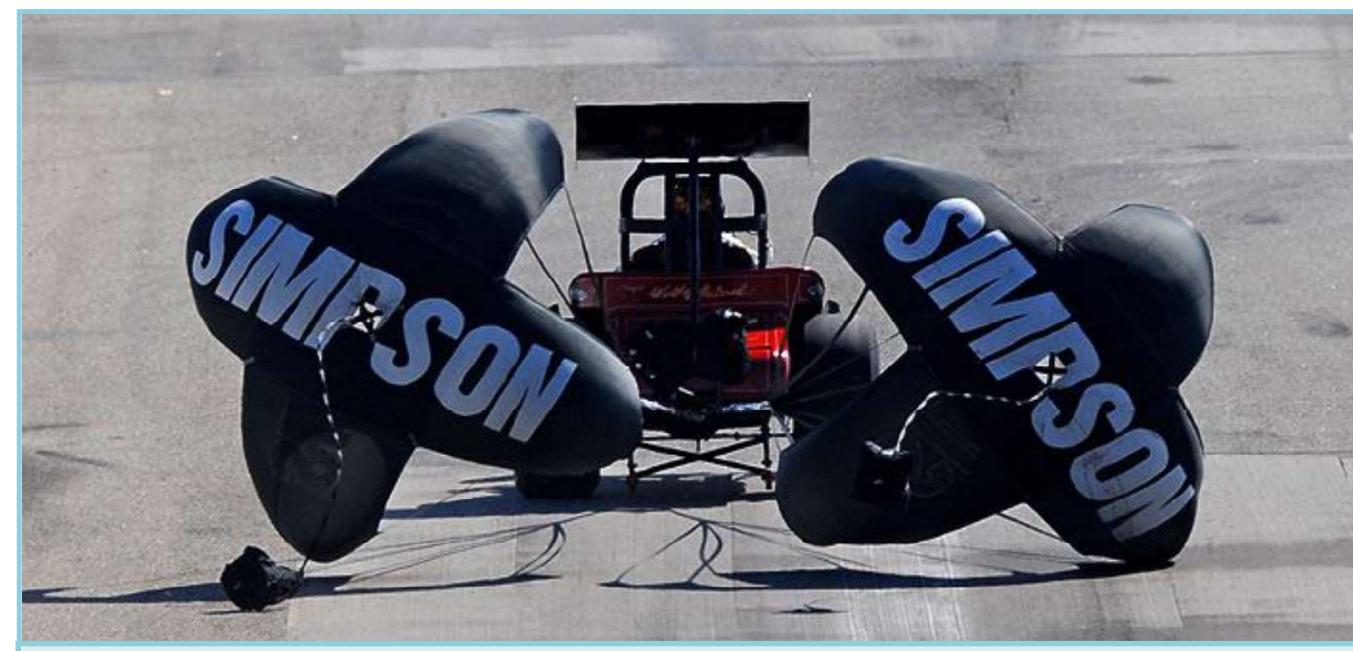

Figure 1: Dragster with its parachutes deployed for stopping. 


\section{MOTIVATION}

Drag is a fundamental force that affects all objects that encounter some sort of motion; whether it is primary motion or external motion. To realize how drag affects objects is useful to science because drag forces affect a majority of objects we use on a daily basis. The force applied to a hand held outside a speeding car is a simplistic example of drag. Scientist and engineers can use the knowledge about drag forces to solve many problems. Some examples of problems involving drag forces are:

- Fuel efficiency in motor vehicles

- Aviation

- Degradation of bridge pilings in a channel

- Productive drag (parachutes)

Drag forces are often associated with inefficiency; but in some cases drag is a necessary tool used by engineers and scientist. The question listed in the problem statement is an example of where drag forces are absolutely crucial. A parachute utilizes drag forces to slow down high speed objects such a racers, aircraft, and even people.

\section{MATHEMATICAL DESCRIPTION AND SOLUTION APPROACH}

Drag force $\left(F_{D}\right)$ on an object depends on the following quantities:

- Velocity $(v)$

- Fluid Density $(\rho)$

- $\operatorname{Viscosity~}(\mu)$

- Area affected by motion, $(A)$ 
Deriving a relationship between these five variables might take numerous hours and a lot of experimental data. Instead, all five quantities can be expressed in the fundamental dimensions of mass $(M)$, length $(L)$, and time $(T)$ and Buckingham's $\pi$ Theorem can be used to balance the dimensions properly (Buckingham, 1914). See Table 2 in Appendix A for unit conversions.

\section{$\underline{\text { BUCKINGHAM'S } \pi \text { THEOREM }}$}

Buckingham's $\pi$ Theorem says two things (Singer et. al., 1998):

1. If an equation is dimensionally homogeneous it can be reduced to a relationship among a complete set of dimensionless products.

2. The number of independent dimensionless products required to express a relationship between the variables in any phenomenon is equal to the number of derived variables involved $n$, minus the number of dimensions $m$, or primary variables, in which these quantities may be measured, i.e. the number of $\pi$ terms is $n-m$.

Step 1: $\quad$ Assume $f\left(F_{D}, v, \rho, \mu, A\right)=0$ for some function $f$. Note that the five quantities $F_{D}, v, \rho, \mu$, and $A$ may be expressed in three fundamental dimensions $L, M$, and $T$. Buckingham's $\pi$ theorem says that the drag force $F_{D}$ can be expressed in terms of $n-m=5-3=2$ dimensionless quantities, i.e. $f(v, \rho, \mu, A)=g\left(\pi_{1}, \pi_{2}\right)$ for dimensionless $\pi_{1}$ and $\pi_{2}$.

Step 2: $\quad$ From Table 1 in the Appendix note that,

$$
F_{D}=\left[\frac{M L}{T^{2}}\right], \quad v=\left[\frac{L}{T}\right], \quad \rho=\left[\frac{M}{L^{3}}\right], \quad \mu=\left[\frac{M}{L T}\right], \quad A=\left[L^{2}\right]
$$


Step 3: $\quad$ Select primary repeating variables, such that all fundamental dimensions are represented. Choose $v, \rho, \mu$ for instance.

Step 4: $\quad$ Let the dimensionless quantities equal the product of powers of the reoccurring quantities from Step 3 and the non-reoccurring quantities. This means

$$
\left\{\begin{array}{l}
\pi_{1}=v^{a} \rho^{b} \mu^{c} F_{D} \\
\pi_{2}=v^{d} \rho^{e} \mu^{f} A
\end{array}\right.
$$

Step 5: $\quad$ For $\pi_{1}$ and $\pi_{2}$ to be dimensionless,

$$
\left\{\begin{array}{l}
\pi_{1}=\left[\frac{L}{T}\right]^{a}\left[\frac{M}{L^{3}}\right]^{b}\left[\frac{M}{L T}\right]^{c}\left[\frac{M L}{T^{2}}\right]=L^{a-3 b-c+1} M^{b+c+1} T^{-a-c-2}=L^{0} M^{0} T^{0} \\
\pi_{2}=\left[\frac{L}{T}\right]^{d}\left[\frac{M}{L^{3}}\right]^{e}\left[\frac{M}{L T}\right]^{f}\left[L^{2}\right]=L^{d-3 e-f+2} M^{e+f} T^{-d-f}=L^{0} M^{0} T^{0}
\end{array}\right.
$$

Step 6: $\quad$ Solve the two systems of equations in Step 5:

$$
\left[\begin{array}{cccc}
1 & -3 & -1 & -1 \\
0 & 1 & 1 & -1 \\
-1 & 0 & -1 & 2
\end{array}\right] \sim\left[\begin{array}{cccc}
1 & 0 & 0 & 0 \\
0 & 1 & 0 & 1 \\
0 & 0 & 1 & -2
\end{array}\right]
$$

and

$$
\left[\begin{array}{cccc}
1 & -3 & -1 & -2 \\
0 & 1 & 1 & 0 \\
-1 & 0 & -1 & 0
\end{array}\right] \sim\left[\begin{array}{cccc}
1 & 0 & 0 & 2 \\
0 & 1 & 0 & 2 \\
0 & 0 & 1 & -2
\end{array}\right]
$$

so $a=0, b=1, c=-2$, and $f=-1 / 2$. So the equations in Step 4 become

$$
\left\{\begin{array}{l}
\pi_{1}=\frac{F_{D} \rho}{\mu^{2}} \\
\pi_{2}=\frac{v^{2} \rho^{2} A}{\mu^{2}}
\end{array}\right.
$$


Step 7: $\quad$ Consider the following transformations of $\pi_{1}$ and $\pi_{2}$ :

$$
C_{D}=\frac{\pi_{1}}{2 \pi_{2}}=\frac{F_{D}}{\frac{1}{2} \rho A v^{2}}
$$

and

$$
R e=\sqrt{\pi_{2}}=\frac{v \rho \sqrt{A}}{\mu} .
$$

Since $g\left(\pi_{1}, \pi_{2}\right)=0$ governs the drag forces, this can be rewritten as:

$$
\tilde{g}\left(C_{D}, R e\right)=0 .
$$

In fact, $C_{D}$ is called the Drag Coefficient and $R e$ is known as the Reynolds $\underline{\text { Number }}$. Note that both $C_{D}$ and $R e$ are dimensionless as $\pi_{1}$ and $\pi_{2}$ are dimensionless. It is important to remark that the drag force function can be expressed by the definition of $C_{D}$, i.e.

$$
F_{D}=\frac{1}{2} \rho v^{2} C_{D} A
$$

Now that the drag force equation is derived, we can solve a problem to see the effects of drag forces.

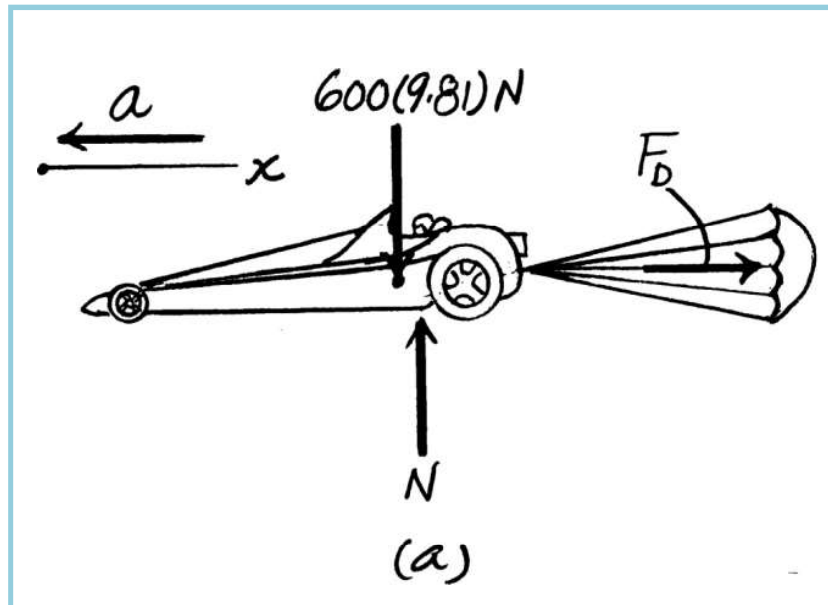

Figure 2: Free body diagram describing forces at work on the dragster 
Consider the question asked in the problem statement:

"A $600 \mathrm{~kg}$ dragster is traveling with a velocity of $125 \mathrm{~m} / \mathrm{s}$ when the engine is shut off and the braking parachute is deployed. If air resistance imposed on the dragster due to the parachute is given by the function $F_{D}=\left(6000+0.9 v^{2}\right) \mathrm{N}$, where $v$ is in $\mathrm{m} / \mathrm{s}$ determine the time required for the dragster to come to rest. Also find the distance traveled in that time period."

Let's rephrase the question to :

"Assume the parachute of the $600 \mathrm{~kg}$ dragster opens at time $t_{0}=0 \mathrm{~s}$ at position $p\left(t_{0}\right)=0 \mathrm{~m}$ with velocity $v\left(t_{0}\right)=125 \mathrm{~m} / \mathrm{s}$ and coasts to rest at time $t_{1}$ at position $p\left(t_{1}\right)$ when velocity $v\left(t_{1}\right)=0 \mathrm{~m} / \mathrm{s}$ because of a drag force $F_{D}=\left(6000+0.9 v(t)^{2}\right) N$. Find $t_{1}$ and $p\left(t_{1}\right) . "$

First, it is beneficial to draw a free body diagram describing the forces acting on the object (see Figure 2). Notice that the drag force is acting against the cars motion. Since the drag force is given, we will not have to use the drag force equation previously derived.

To solve this, first consider Newton's second law of motion:

$$
\begin{aligned}
\sum F_{x} & =m a_{x} \\
6,000+0.9 v^{2} & =600(-a) \\
a & =-\left(10+\frac{3}{2,000} v^{2}\right) \mathrm{m} / \mathrm{s}^{2}
\end{aligned}
$$


which yields an expression for the acceleration of the dragster in terms of its velocity. Noting that acceleration is the derivative of velocity with respect to time, we may use integration and the chain rule to find the time at $t_{1}$ (see Appendix B, Section A for details):

$$
t_{1}=\int_{0}^{t_{1}} d t=\int_{v(0)}^{v\left(t_{1}\right)} \frac{d v}{a}=\int_{125}^{v\left(t_{1}\right)} \frac{d v}{-\left(10+\frac{3}{2,000} v^{2}\right)}=10 \sqrt{\frac{2}{3}}\left[\tan ^{-1}\left(\frac{5}{4} \sqrt{\frac{3}{2}}\right)-\tan ^{-1}\left(\frac{1}{100} \sqrt{\frac{3}{2}} v\left(t_{1}\right)\right)\right]
$$

The velocity is $0 \mathrm{~m} / \mathrm{s}$ when the dragster comes to rest, so

$$
t_{1}=10 \sqrt{\frac{2}{3}}\left[\tan ^{-1}\left(\frac{5}{4} \sqrt{\frac{3}{2}}\right)-\tan ^{-1}(0)\right] \approx 8.101 \mathrm{~s}
$$

To find the distance traveled in those 8.101 seconds, simply express $v(t)$ in terms of $t$ and integrate, i.e.

$$
\begin{aligned}
p\left(t_{1}\right)=\int_{0}^{t_{1}} v(t) d t & =\int_{0}^{t_{1}} 100 \sqrt{\frac{2}{3}} \tan \left(\tan ^{-1}\left(\frac{5}{4} \sqrt{\frac{3}{2}}\right)-\frac{1}{10} \sqrt{\frac{3}{2}} t\right) d t \\
& =\frac{1000}{3} \ln \left(\frac{107}{32}\right) \approx 402.364 \mathrm{~m} .
\end{aligned}
$$

(See Appendix B, Section B and C for further details).

\section{DISCUSSION}

This project yielded several results. Drag forces have many applications such as fuel efficiency in production vehicles, aviation, degradation of bridge pilings, and parachutes. 
Buckingham's $\pi$ Theorem showed that the equation governing drag forces is dependent on the viscosity and velocity of the passing air and the shape and area of the material factor.

In the dragster problem, after the parachute opened it took 8.101 seconds for the car to come to a complete stop. From experience, anything around 10 seconds would have seemed reasonable. The car also rolled $402.364 \mathrm{~m}$ in those 8.101 seconds which is approximately $1,320.09 \mathrm{ft}$. It is important to note that this is a long distance to travel in such a short amount of time. In the field this has very visible repercussions. This method can be used to determine how much track is needed for racecars to safely come to a stop.

\section{CONCLUSION AND RECOMMENDATIONS}

This project defined what a drag force is, derived an equation for drag force and gave examples of those drag forces. Drag is a force that opposes motion due to an objects shape and area, material, and speed. With the help of dimensional analysis and Buckingham's $\pi$ Theorem, this equation was derived as

$$
F_{D}=\frac{1}{2} \rho v^{2} C_{D} A
$$

An example involving the drag forces on the parachute of a dragster revealed the time and distance necessary for the racecar to reach a full stop by air resistance alone. For future projects, it is recommended that problems fully utilize the drag equation instead of providing the drag forces in the question. 


\section{NOMENCLATURE}

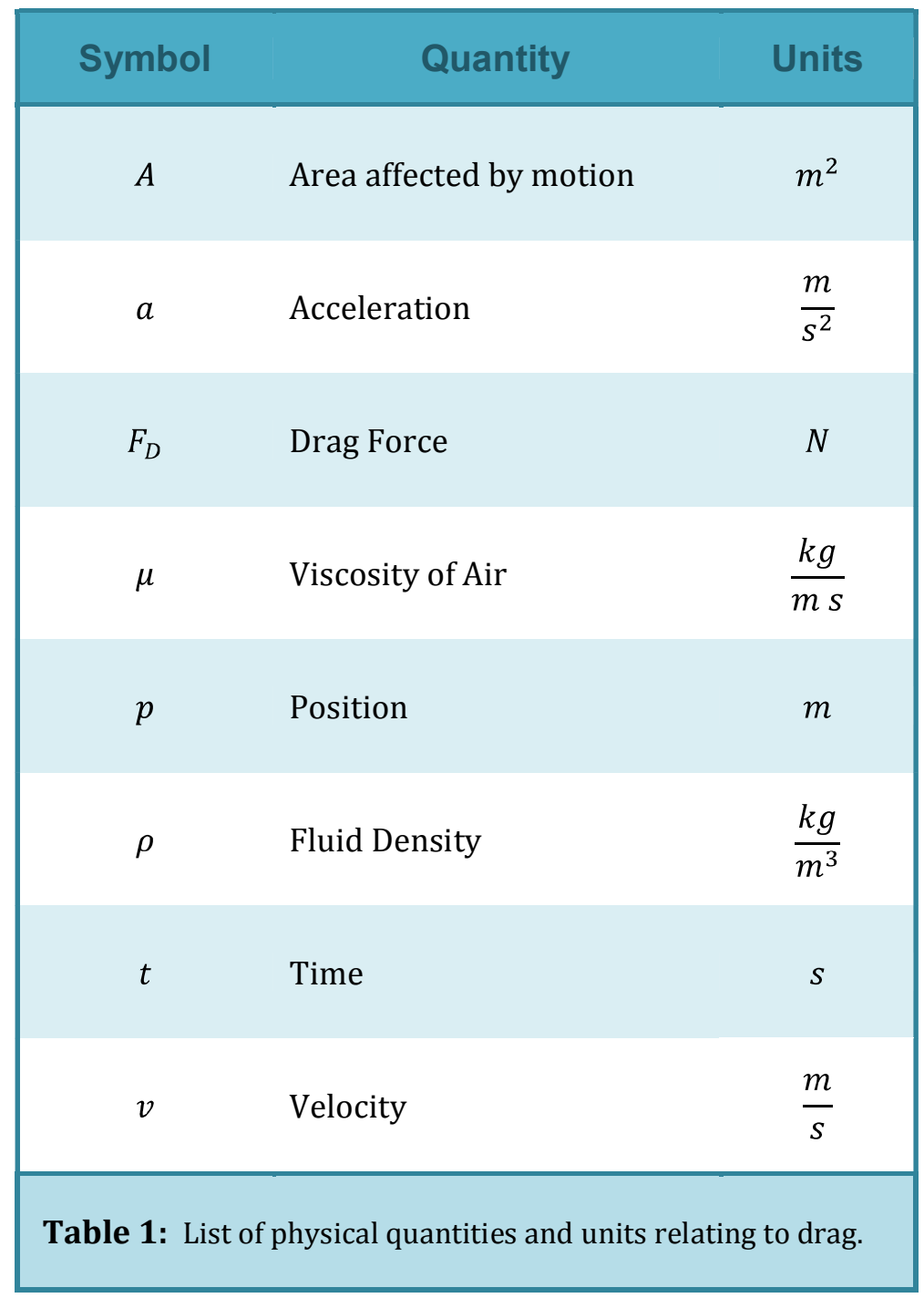




\section{REFERENCES}

\section{BOOKS AND ARTICLES}

Buckingham, E. (1914). "On physically similar systems; illustrations of the use of dimensional equations". Phys. Rev. 4: 345-376.

Singer, Arbocz, Weller. (1998). Buckling Experiments: Experiments in Thin-Walled Structures. Volume 1: Basic concepts, columns, beams and plates. John Wiley \& Sons, Inc. pp.218-219

\section{Web RESOURCES}

Bengtson, H. (Jun 1, 2010). "Drag Force for Fluid Flow Past an Immersed Object.” Bright Hub: http://www.brighthub.com/engineering/civil/articles/58434.aspx

Benson, T. (Mar. 16, 2010). “Shape Effects on Drag.” NASA Webpage: http://www.grc.nasa.gov/WWW/K-12/rocket/shaped.html 


\section{APPENDIX A - TABLES}

\begin{tabular}{|clc|}
\hline Symbol & \multicolumn{1}{c|}{ Quantity } & Dimensions \\
\hline$A$ & Area affected by motion & $L^{2}$ \\
$F_{D}$ & Drag Force & $M L T^{-2}$ \\
$\mu$ & Viscosity of Air & $M L^{-1} T^{-1}$ \\
$\rho$ & Fluid Density & $M L^{-3}$ \\
$V$ & Velocity & $L T^{-1}$ \\
\hline \multicolumn{2}{|c|}{ Table 2: } & List of physical quantities and the fundamental \\
& dimensions they are measured in. \\
\hline
\end{tabular}

\section{Shape}

Drag Coefficient

Rough sphere $(R e=1,000,000)$ 0.4

Smooth sphere $(R e=1,000,000)$

0.1

Turbulent flow parallel to a flat plate $(R e=1,000,000)$ 0.005

Laminar flow parallel to a flat plate $(R e=1,000,000)$ 0.001

Hummer H2 (2003)

Toyota Prius (2004 - 2009) 0.26

Bullet at subsonic velocity 0.295

Table 3: Standard drag coefficients (Bengtson, 2010) 


\section{APPENDIX B - COMPUTATION DETAILS}

A. Calculating a relationship between time $t_{1}$ and velocity $v\left(t_{1}\right)$ through the acceleration equation:

$$
\begin{aligned}
t_{1}=\int_{0}^{t_{1}} d t & =\int_{v(0)}^{v\left(t_{1}\right)} \frac{d v}{a} \\
& =\int_{125}^{v\left(t_{1}\right)} \frac{d v}{-\left(10+\frac{3}{2,000} v^{2}\right)} \\
& =-\left(\sqrt{\left.\frac{2,000}{3}\right)} \int_{125}^{v\left(t_{1}\right)} \frac{\left(\sqrt{\frac{3}{2,000}} d v\right)}{(\sqrt{10})^{2}+\left(\sqrt{\frac{3}{2,000}} v\right)^{2}}\right. \\
& =-\left.\frac{1}{\sqrt{10}}\left(\sqrt{\frac{2,000}{3}}\right)\left[\tan ^{-1}\left(\frac{\left.\sqrt{\frac{3}{2,000}} v\right)}{\sqrt{10}}\right)\right]\right|_{125} ^{v\left(t_{1}\right)} \\
& =10 \sqrt{\frac{2}{3}}\left[\tan ^{-1}\left(\frac{5}{4} \sqrt{\frac{3}{2}}\right)-\tan ^{-1}\left(\frac{1}{100} \sqrt{\frac{3}{2}} v\left(t_{1}\right)\right)\right]
\end{aligned}
$$

B. Solving for velocity in terms of time $t_{1}$ :

$$
\begin{aligned}
& t_{1}=10 \sqrt{\frac{2}{3}}\left[\tan ^{-1}\left(\frac{5}{4} \sqrt{\frac{3}{2}}\right)-\tan ^{-1}\left(\frac{1}{100} \sqrt{\frac{3}{2}} v\left(t_{1}\right)\right)\right] \\
& \frac{1}{10} \sqrt{\frac{3}{2}} t_{1}=\tan ^{-1}\left(\frac{5}{4} \sqrt{\frac{3}{2}}\right)-\tan ^{-1}\left(\frac{1}{100} \sqrt{\frac{3}{2}} v\left(t_{1}\right)\right) \\
& \tan ^{-1}\left(\frac{1}{100} \sqrt{\frac{3}{2}} v\left(t_{1}\right)\right)=\tan ^{-1}\left(\frac{5}{4} \sqrt{\frac{3}{2}}\right)-\frac{1}{10} \sqrt{\frac{3}{2}} t_{1} \\
& \frac{1}{100} \sqrt{\frac{3}{2}} v\left(t_{1}\right)=\tan \left[\tan ^{-1}\left(\frac{5}{4} \sqrt{\frac{3}{2}}\right)-\frac{1}{10} \sqrt{\frac{3}{2}} t_{1}\right] \\
& v\left(t_{1}\right)=100 \sqrt{\frac{2}{3}} \tan \left[\tan ^{-1}\left(\frac{5}{4} \sqrt{\frac{3}{2}}\right)-\frac{1}{10} \sqrt{\frac{3}{2}} t_{1}\right]
\end{aligned}
$$


C. Solving for position when time $t_{1}=10 \sqrt{\frac{2}{3}}\left[\tan ^{-1}\left(\frac{5}{4} \sqrt{\frac{3}{2}}\right)\right] \approx 8.101$ :

$$
\begin{aligned}
p\left(t_{1}\right) & =\int_{0}^{t_{1}} v(t) d t \\
& =\int_{0}^{t_{1}} 100 \sqrt{\frac{2}{3}} \tan \left(\tan ^{-1}\left(\frac{5}{4} \sqrt{\frac{3}{2}}\right)-\frac{1}{10} \sqrt{\frac{3}{2}} t\right) d t \\
& =\left(-10 \sqrt{\frac{2}{3}}\right)\left(100 \sqrt{\frac{2}{3}}\right) \int_{0}^{t_{1}} \tan \left(\tan ^{-1}\left(\frac{5}{4} \sqrt{\frac{3}{2}}\right)-\frac{1}{10} \sqrt{\frac{3}{2}} t\right)\left(-\frac{1}{10} \sqrt{\frac{3}{2}} d t\right) \\
& =\frac{2000}{3} \ln \left|\cos \left(\tan ^{-1}\left(\frac{5}{4} \sqrt{\frac{3}{2}}\right)-\frac{1}{10} \sqrt{\frac{3}{2}} t\right)\right|_{0}^{t_{1}} \\
& =\frac{2000}{3} \ln \left|\frac{\cos \left(\tan ^{-1}\left(\frac{5}{4} \sqrt{\frac{3}{2}}\right)-\frac{1}{10} \sqrt{\frac{3}{2}} t_{1}\right) \mid}{\cos ^{-1}\left(\tan ^{-1}\left(\frac{5}{4} \sqrt{\frac{3}{2}}\right)\right)}\right| \frac{2000}{3} \ln \left|\frac{1}{4} \sqrt{\frac{107}{2}}\right| \\
& \approx \frac{2000}{3} \ln \left|\frac{\cos \left(\tan ^{-1}\left(\frac{5}{4} \sqrt{\frac{3}{2}}\right)-\tan ^{-1}\left(\frac{5}{4} \sqrt{\frac{3}{2}}\right)\right) \mid}{32}\right| \\
& =\frac{107}{364} \mid
\end{aligned}
$$




\section{APPENDIX C - OTHER DRAG RESOURCES}
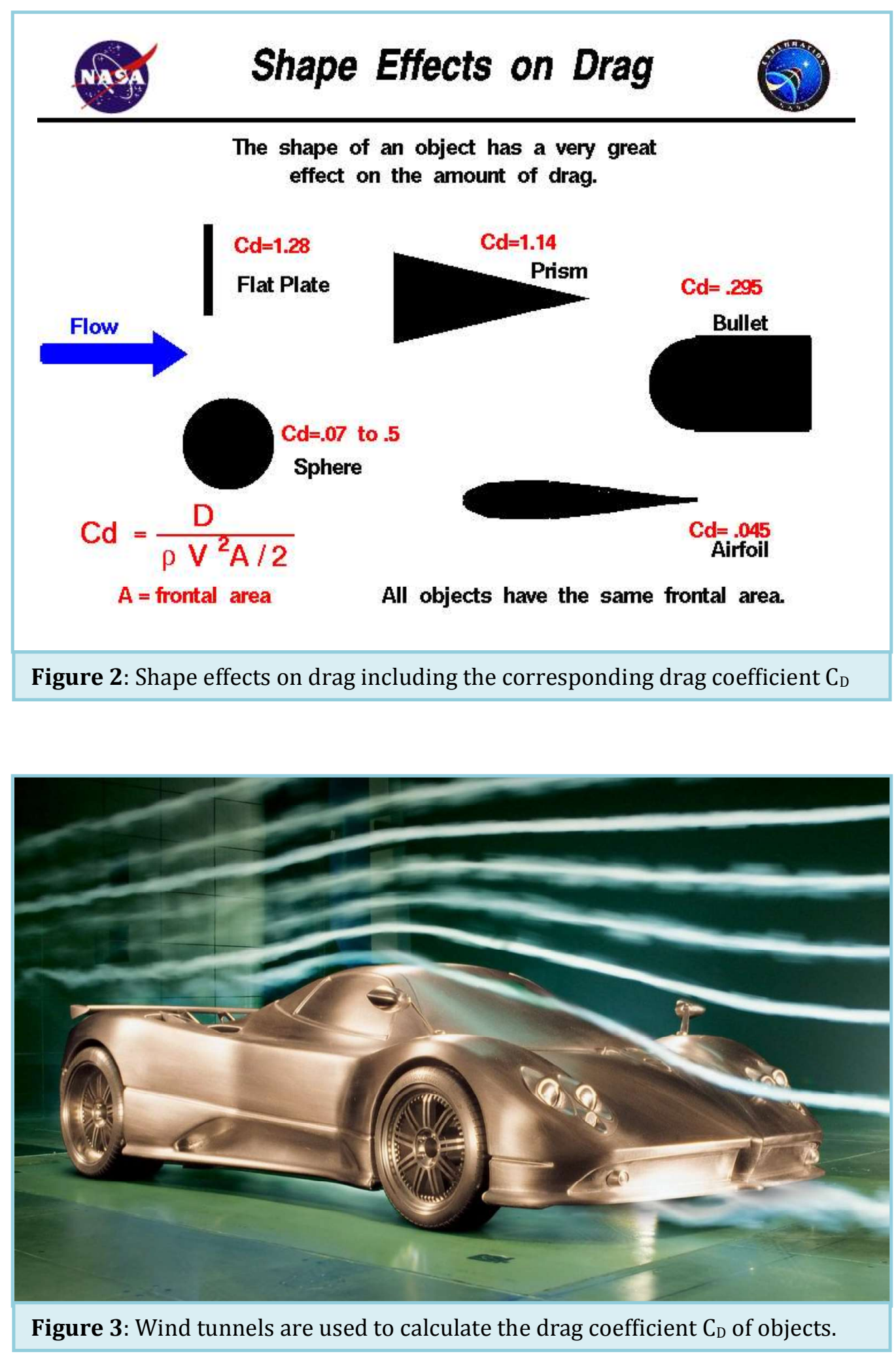